\title{
The theory on thing's limits. Part 1: The norm of identifying truth
}

\author{
Jian DING ${ }^{*}$ \\ (Retired, Integrated Electronic Systems Lab Co. Ltd., Jinan 250100, China)
}

\begin{abstract}
Things refer to the objects and phenomena in reality. Limits refer to the truths and the processes of approaching them. Then there is the principle of inertia, which is characterized by continuity, and a necessary condition for reasoning in reality. Therefore, things, limits and inertia are the three elements that constitute "The Theory on Thing's Limits". The truth must have absoluteness and immutability, does not exist in reality, and belongs to the category of metaphysics. The characteristic of the truths is that they cannot be proved by empirical methods, and can only be gradually approached by repeated practices. Based on this, this article has established the norm for identifying truth. It not only has achieved the unity of opposites of all knowledge, but can also according to have the characteristic of continuity on the change of objective things make bidirectional reasoning under the premise of mutual restrictions. From the quantitative change in the real space has gone deep into the qualitative change of ideal realm, it extends the philosophy of materialism to the category of metaphysics. As a result, the true nature of metaphysics has been restored. That is to say, it has neither divorced from practices, nor just observed objective things with a one-sided, isolated and static way of thought. The theory is applicable to all academic categories, and it is to provide an effective method for testing authoritative theories, clarifying chaos, and deriving new knowledge. Finally, according to the theory and norm therein, combining with the objective status quo, this article briefly has analyzed three examples and pointed out the crux of Einstein's special relativity.
\end{abstract}

Key words: truth; things; limits; inertia; photons; metaphysics; special relativity

PACS: 01.70.+w; 11.10.Cd; 02.90.+p; 03.30.+p

* Corresponding author introduction: Jian DING, Male, Retired, metaphysics and physics. E-mail: jiandus@163.com 


\section{Introduction}

In reality, everything is always in the process of change, and the only constant is the change itself. As the argument of this article, this is a truth. That is to say, truth must have absoluteness and immutability, does not exist in reality, and belongs to the category of metaphysics. Therefore, truth cannot rely on some so-called perfect experiments to be proved, but should be in difficult and embarrassing environments gradually to modify the one-sided views to approach it. ${ }^{[1]}$

This article is the first part of "The Theory on Thing's Limits". As far as this theory is concerned, it aims to study the objective laws between "being" and "non-being". Things refer to the objects and phenomena in reality. Limits refer to the truths and the processes of approaching them. Then there is the following axiom: the change of anything has continuity. This axiom can also be called as the principle of inertia: the development of anything has a tendency to maintain its state just a moment ago. This is the basis of reasoning in reality, and can also be called as the necessary condition. This, of course, is also a necessary condition for causality to come into being. The evolution of everything in the universe, which is precisely a process from quantitative change to qualitative change based on that. Therefore, things, limits and inertia are the three elements that constitute "The theory on thing's limits".

The principle of seeking limit in mathematics, gradually approaching the limit value by the way of infinite subdivision, it is abstracted from the physical processes of identifying the truths. Among them, the truths correspond to the limit value in mathematics, they all do not exist in reality, and belong to the category of metaphysics. Therefore, the processes of identifying truth can only be gradually approached by repeated practices. When the difference between the obtained conclusion and the truth can be an arbitrarily small value, according to the above-mentioned inertia principle, this difference can be made up by the arbitrarily small value of maintaining inertia. This means that the truth has been identified. As a result, we can break through the bondage of finite thought, from the quantitative change in the real space have gone deep into the qualitative change of ideal realm, and extend the philosophy of materialism to the category of metaphysics.

\section{Restore the true nature of metaphysics}

Drawing on Aristotle's definition for reference and distinguishing different definition domains according to whether it exists in reality, all knowledge can be divided into three parts: natural science 
(materialism), metaphysics (idealism) and mathematics. And mathematics has helped us to break through the bondage of finite thought by the way of infinite subdivision, from the quantitative change in real space has gone deep into the qualitative change of ideal realm. That is to say, mathematics runs through two different domains of definition, not only has achieved the unity of opposites of all knowledge, but can also help us to make bidirectional reasoning under the premise of mutual restrictions according to the change of objective things. Therefore, it is vital to determine different domains of definition reasonably in the process of reasoning. Seeing the essence through the phenomena, as a result, the true nature of metaphysics has been restored. This means that it has neither divorced from practices, nor just observed objective things with a one-sided, isolated and static way of thought. ${ }^{[2]}$

The word "metaphysics" was translated from Japanese into Chinese. Among them, English's "physics" has originated from the ancient Greek, and the original meaning is "natural"; while the prefix "meta" contains the implications of "behind, support and origin". So it is also reasonable to translate "metaphysics" into "the origin of natural laws". Its "existence" is only in order to the existence of the natural science, as a support or an origin behind that, but does not exist in reality. Aristotle defined it as "first philosophy", also known as "theology". Yan Fu (AD 1854-1921), a Chinese scholar, also once translated it into "dark learning". This meant that in the philosophical category, any reference to terms, such as the first philosophy, theology or dark learning, should be considered to refer to metaphysics. The contents contained in metaphysics, such as noumenon, axiom or postulation, absolute motion, etc., all can be called as the truths. They all have to have absoluteness and immutability, and do not exist in reality. That is to say, their "existences" only in order to the existence of the natural science and related mathematics.

\section{The norm for identifying truth}

The so-called bidirectional reasoning is that based on the above-mentioned principle of inertia, the reasoning main body should be under the restrictions of relevant truths and objective facts, and starting from a certain truth, through objective facts that have continuity with it, and then gradually approaching or confirming a hypothesis. As for backward reasoning, it is to start from objective facts and gradually approach or confirm a hypothesis. If only as far as the norm is concerned, it has been included in the above-mentioned reasoning main body. In the above-mentioned reasoning processes, 
any hypothesis that can be confirmed by the means of reality is an objective fact. If the hypothesis can only be approached gradually by objective facts, and the difference between the two has reached an arbitrarily small value, then should also reflect on those objective facts related to the reasoning process, and when confirming the hypothesis, look at whether they have done all out. If it is, then according to the above-mentioned principle of inertia, this difference value can be made up by the arbitrarily small additional value in the "tendency to maintain its state just a moment ago". As a result, the hypothesis can be identified as a truth. Otherwise, the reasoning fails, this hypothesis is not valid, and it needs to be reflected on. This is the norm of how to identify truth, which is established by "The theory on thing's limits" in this article.

For example, how to identify the point positions of natural numbers on the number axis is a problem that every student will encounter. Take the natural number "1" for example, only the point position of its noumenon is absolutely accurate. But if you use the actual means to confirm, no matter how accurately to approach it, the point position you determine is either less than $1-10^{-\mathrm{n}}$, or greater than $1+10^{-\mathrm{n}}$ ( $\mathrm{n}$ is a natural number that can satisfy the argument). And they all seem to try their best to show that there is an absolutely accurate point position between the two sets of points. This is because the noumenon of natural number and its absolute point position on the number axis do not exist in reality, and can only be approached as much as possible.

But people usually do not reflect on the above facts, instead, they determine a point position on the number axis with reasonable accuracy, and mark it with the natural number "1". After that, whenever people see this point position on the number axis to be marked as a natural number "1", a consensus of absolute no error will be reached between them. Usually they don't go to reflect on that the consensus generated in their subconsciousness can only be the noumenon of the natural number "1", while this noumenon and its absolute point position on the number axis do not exist in reality. Similarly, the same is true to identify the point positions of other natural numbers on the number axis.

Thus it can be seen that metaphysics is indispensable in people's daily life, and used by everyone, just they do not deliberately reflect on. Otherwise, such a consensus of absolute no error would not be reached between them. And once an error is produced, a minimal error may result in wide divergence, the reason therein is well-known, so there is no need to say more about it. 


\section{The value c of light speed in vacuum}

The value $\mathrm{c}$ of light speed in vacuum is a physical constant. In October 1983, at the 17th General Conference of Weights \& Measures (CGPM) held, c=299792458 (m/s) was specified as the value of light speed in vacuum after the new definition of the length unit "m" was voted through. It was an exact value with an uncertainty equaling 0 , and an absolute accurate reference value. This showed that there were many mainstream scientists in the world had already confirmed by a large number of scientific experiments that the highest speed existed in the universe, and the measurement error on it had been very small. So, in order to define this limit value of the speed in the universe, the value $\mathrm{c}$ of light speed in vacuum, they even intended the risk of fine-tuning the unit metering length.

This was a very wise decision. The only regret was that these mainstream scientists, perhaps they themselves did not really understand that the speed of light $\mathrm{c}$ in a vacuum was an absolute motion beyond real space. In reality, no matter what experimental means were used, the experimental results obtained were only able to gradually approach, but not able to equal to this limit value of the speed in the universe. Therefore, as an absolute frame of reference, the value c of light speed in vacuum should be just higher an arbitrarily small value than the highest light speed in reality.

It was precisely because Einstein failed to comprehend this key of leading to the truth, thereby fell into the dilemma that the light speed in reality was equal to the c. Although he put the value $\mathrm{c}$ of light speed in vacuum as a base point to use repeatedly in his special relativity, but because of his vagueness of concept, and resolutely "gives no basis for the introduction of the concept of absolute motion" ${ }^{[3]}$, so he had to offend this truth, that was, the absoluteness of simultaneity, and went astray. It seemed that he was forced to have no choice but to do this, actually which was because he didn't know what a truth was, so fearless.

In fact, Hendrik Antoon Lorentz and Jules Henri Poincaré had also encountered such a problem before he did, but they not dared to rashly offend Newton's concept of absolute space-time. At that time, Poincaré also knew that the postulation could never be verified directly by experience ${ }^{[4]}$, and seemed to have reached a state where the $\mathrm{c}$ was a truth by default. It can be seen that authoritative theories must pass the test of truth, and then gradually gain public recognition. Although this process may be long, it is insignificant compared to eternal truth. 


\section{The absoluteness of simultaneity}

As truth, the absoluteness of simultaneity can be confirmed according to the above-mentioned norm for identifying truth. However, Einstein might not understand that truth could not be proved by a so-called perfect experiment, and only be gradually approached through repeated practice. Therefore, Einstein was in order to prove his principle of relativity to use the Lorentz coordinate transformation to replace with the Galileo's coordinate transformation, which was actually at the expense of truth (absoluteness of simultaneity). ${ }^{[2]}$

On November 28, 1919, in his article in The Times, he said ${ }^{[5]}$ that "It became clear that to speak of the simultaneity of two events had no meaning except in relation to a given coordinate system, and that the shape of measuring devices and the speed at which clocks move depend on their state of motion with respect to the coordinate system." Its meaning can be interpreted in this way that if there are two events A and B with simultaneity in a given coordinate system, then when you have only subdivided this coordinate system into two coordinate systems and the events A and B are in both respectively, their original simultaneity would become no meaning. In other words, as long as coordinate systems are redefined in the same space-time, the original simultaneous events would be not simultaneously.

This is exactly the crux of Einstein's special relativity ${ }^{[4]}$. Regarding this, no matter his supporters or opponents, there is a consensus in the sub-consciousness deep inside each one of their hearts, that is, there is a doubt about this, but cannot be verified by the means in reality. From this resulting paradoxes ${ }^{[6]}$, such as the so-called time dilation and length contraction effects, and a photon without mass but with energy, and so on, have been popular for more than a hundred years. Reflecting on these arguments have a common feature, that is, all the objective facts related to them are doing their best to point out that these arguments are paradoxes.

This example tells us that in the process of reasoning, if you offend a certain truth, it must be carefully reflected on. Because it can help to find human errors in the reasoning process. Otherwise, even though all of your reasoning processes are correct ${ }^{[4]}$, the result would become a paradox, and not be supported by bjective facts. If these methods are used respectively to identify the absoluteness of simultaneity, and the so-called time dilation and length contraction effects, it is possible to deepen the understanding how to identify between the truth and paradox. 


\section{Conclusion}

This article "The theory on thing's limits" is applicable to all fields of knowledge, and it is an effective method for testing authoritative theories, clarifying chaos, and deriving new knowledge. The truth must have absoluteness and immutability, does not exist in reality, and belongs to the category of metaphysics. The characteristic of the truths is that they cannot be proved by empirical methods, and can only be gradually approached by repeated practices.

Things refer to the objects and phenomena in reality. Limits refer to the truths and the processes of approaching them. Then there is the principle of inertia: the development of anything has a tendency to maintain its state just a moment ago. That is the continuity, which is a necessary condition for reasoning in reality. Therefore, things, limits and inertia are the three elements that constitute "The theory on thing's limits".

This article is based on the relationship between things and truths, and with the help of the principle of inertia, has established the norm for identifying truth. It not only has achieved the unity of opposites of all knowledge, but can also according to have the characteristic of continuity on the change of objective things make bidirectional reasoning under the premise of mutual restrictions. From the quantitative change in the real space has gone deep into the qualitative change of ideal realm, it extends the philosophy of materialism to the category of metaphysics. As a result, the true nature of metaphysics has been restored. This means that it has neither divorced from practices, nor just observed objective things with a one-sided, isolated and static way of thought.

After that, according to the theory on thing's limits and the norm for identifying truth therein, combining with the practical applications, after a brief analyses, it can be summarized as follows:

1. Through identifying the point positions of natural numbers on the number axis and the process of defining the value c of light speed in vacuum, with the help of these two examples, it can be illustrated that metaphysics is indispensable, and used by everyone.

2. As a physical constant, the value $\mathrm{c}$ of light speed in vacuum is an absolutely accurate reference value. It is the limit value of the speed in the universe, and is an absolute motion, which must be just higher an arbitrarily small value than the highest light speed in reality. It is precisely because Einstein failed to comprehend this key of leading to the truth, thereby he fell into the dilemma that the light speed in reality was equal to the c. 
3. The absoluteness of simultaneity, it is the truth that can be identified according to the norm. Einstein offended this truth, which was exactly the crux of his special relativity. Therefore, such as the so-called time dilation and length contraction effects, and a photon without mass but with energy, and so on, a series of paradoxes were deduced from this. The root cause was that there was the problem with his understanding on absolute motion.

\section{References}

[1] Jian DING. The Research of Using Truth to Restrict Authoritative Theories. Journal of Philosophy and Ethics. 2020; 2(1): 43-50.

[2] Jian D. Piercing the veil of modern physics: part 2 \& philosophy. Physics \& Astronomy International Journal. 2018; 2(2):136-144. DOI: 10.15406/paij.2018.02.00075

[3] Albert Einstein. Relativity [A]. Fang Z Q, Han W B, He W G. Out of My Late Years by Albert Einstein [M]. Hainan in China: Hainan Press; 2000: 41-41. (in Chinese)

[4] Zhang Y Z. From Newtonian Mecnanics to Special Relaativity. Mechanics in Engineering, 2005, 27(4): 1-6. (in Chinese)

[5] Albert Einstein. What Is the Theory of Relativity? [A]. Xu L Y, Fan D N. The collected works of Einstein (Vol. 1) [C]. Beijing: The Commercial Press; 1976: 110-111. (in Chinese)

[6] Zhang Y Z. Experimental Foundations of Special Relativity [M]. Beijing: Science Press, 1982: 8-12. (in Chinese) 


\title{
物极理论 1: 认定真理的规范
}

\author{
丁健* \\ 积成电子股份有限公司（已退休） 中国济南 250100
}

摘要: 物, 是指现实中的物体和现象。极, 是指真理, 以及接近它们的过程。再 就是惯性原则, 其特征为连续性, 是在现实中推理的必要条件。因此, 事物、极 限和惯性, 就是构成《物极理论》的三要素。真理, 必须具有绝对性和不变性, 在现实中是不存在的, 归属于形而上学的范畴。真理的特点, 就是无法依据实证 的方法予以证明, 只能通过反复地实践而逐步逼近。据此, 本文确立了认定真理 的规范。不仅成就了全部知识的对立统一, 还可以依据客观事物的变化具有连续 性这个特点, 在相互约束的前提下双向推理。从现实空间中的量变一直深入到理 想境界中的质变, 把唯物主义的哲学观拓展到了形而上学的范畴。从而恢复了形 而上学的本来面目。也就是说, 它既不脱离实践, 也不仅仅是用片面、孤立和静 止的思维方式观察客观事物。该理论适用于所有学科, 是为了检验权威理论, 澄 清混沌, 推导新知, 而提供的有效方法。最后, 依据其中的理论和规范, 结合客 观现状, 简要分析了三个实例, 并指出了爱因斯坦狭义相对论的症结所在。

关键词: 真理; 事物; 极限; 惯性; 光子; 形而上学; 狭义相对论

中图分类号：B01；B081.1； N02；O412.1 